OPEN ACCESS

Edited by:

José Lozano,

University of Malaga, Spain

Reviewed by: Reinhard Christoph Dechant,

ETH Zürich, Switzerland Rosa Barrio,

CIC bioGUNE, Spain

${ }^{*}$ Correspondence:

Girish S. Ratnaparkhi girish@iiserpune.ac.in: girish.iiserpune@gmail.com

Specialty section: Signaling,

a section of the journal Frontiers in Cell and Developmental

Biology

Received: 15 April 2021 Accepted: 01 September 2021 Published: 30 September 2021

Citation:

Nayak $P$, Kejriwal $A$ and Ratnaparkhi GS (2021) SUMOylation of Arginyl tRNA Synthetase Modulates the Drosophila Innate Immune

Response.

Front. Cell Dev. Biol. 9:695630. doi: 10.3389/fcell.2021.695630

\section{SUMOylation of Arginyl tRNA Synthetase Modulates the Drosophila Innate Immune Response}

\author{
Prajna Nayak, Aarti Kejriwal and Girish S. Ratnaparkhi* \\ Indian Institute of Science Education and Research (IISER), Pune, India
}

SUMO conjugation of a substrate protein can modify its activity, localization, interaction or function. A large number of SUMO targets in cells have been identified by Proteomics, but biological roles for SUMO conjugation for most targets remains elusive. The multiaminoacyl tRNA synthetase complex (MARS) is a sensor and regulator of immune signaling. The proteins of this 1.2 MDa complex are targets of SUMO conjugation, in response to infection. Arginyl tRNA Synthetase (RRS), a member of the sub-complex II of MARS, is one such SUMO conjugation target. The sites for SUMO conjugation are Lys 147 and 383. Replacement of these residues by $\operatorname{Arg}\left(R R S^{K 147 R, K 383 R}\right)$, creates a SUMO conjugation resistant variant $\left(\mathrm{RRS}^{S C R}\right)$. Transgenic Drosophila lines for RRS ${ }^{W T}$ and RRSSCR were generated by expressing these variants in a RRS loss of function (lof) animal, using the UAS-Gal4 system. The RRS-lof line was itself generated using CRISPR/Cas9 genome editing. Expression of both $R R S^{W T}$ and $R R S^{S C R}$ rescue the $R R S$-lof lethality. Adult animals expressing $R R S^{W T}$ and $R R S^{S C R}$ are compared and contrasted for their response to bacterial infection by gram positive $M$. luteus and gram negative ECC15. We find that $R R S S C R$, when compared to $R R S W T$, shows modulation of the transcriptional response, as measured by quantitative $3^{\prime}$ mRNA sequencing. Our study uncovers a possible non-canonical role for SUMOylation of RRS, a member of the MARS complex, in host-defense.

Keywords: MARS complex, NFkB, signaling, CRISPR, Cas9, ArgRS

\section{INTRODUCTION}

Aminoacyl-tRNA synthetases (ARSs) are ancient, evolutionary conserved enzymes whose primary housekeeping function is to catalyze the aminoacylation of transfer RNAs (tRNAs) (Schimmel and Soll, 1979; Rubio Gomez and Ibba, 2020). In addition to their primary role of charging tRNA, ARSs also have non-canonical, "moonlighting" functions (Guo and Schimmel, 2013; Yao et al., 2014). These secondary functions are driven by modifications to the polypeptide chain by mutations, domain addition or Post-Translational modifiers (PTMs) (Sampath et al., 2004). ARSs are a target of a variety of PTMs, with phosphorylation being studied extensively (Arif et al., 2017). The small ubiquitin-like modifier [SUMO; (Hay, 2005; Geiss-Friedlander and Melchior, 2007)] is one such PTM that targets ARSs. Proteomic studies on a wide range of eukaryotes have suggested (Panse et al., 2004; Golebiowski et al., 2009; Nie et al., 2009; Hendriks and Vertegaal, 2016; Pirone et al., 2017) that at least fourteen of the twenty ARSs are SUMO conjugated (SUMOylated). 
In mammals, nine of the tRNA synthetases (Glu-Pro, Ile, Leu, met, Gln, Lys, Arg, and Asp) are part of a $\sim 1.2 \mathrm{MDa}$ Multi-aminoacyl tRNA Synthetase (MARS) complex, along with three non-ARS components (AIMP1-3) (Khan et al., 2020). In addition to acting as a "depot" or reservoir for tRNA synthetases and facilitating related translational functions, the release of individual components in response to stimulus, both internal and external, regulate the non-canonical functions of these proteins, inclusive of the AIMPs. The released components can be secreted or relocated to a different cellular compartment (Ray and Fox, TIBS, 2007) (Park SG, Kim 2008, PNAS). The MARS complex is now perceived as a hub for many signaling networks within the cell (Park SG, Kim 2008, PNAS). The MARS complex is conserved from insects to mammals, with the Drosophila MARS complex (Kerjan et al., 1994; Havrylenko and Mirande, 2015) containing orthologs of the 11 components seen in mammals.

In an experiment to uncover proteins that are SUMO conjugated in response to infection, our laboratory identified 12 ARSs as potential targets using a quantitative proteomics screen (Handu et al., 2015). The study suggested that SUMOylation of ARSs was a response to immune signaling. Using an in-bacto SUMO conjugation assay (Nie et al., 2009), we validated a subset of Drosophila ARSs as being SUMOylated. Next, we focused our attention on one substrate, namely Arginyl tRNA synthetase (RRS). We determined that K147 and K383 in RRS were the targets of the SUMO machinery and generated transgenic wildtype and SUMO conjugation resistant (SCR) transgenic lines for RRS using a combination of CRISPR Cas9 genome editing and UAS-Gal4 system. A comparison of the transcriptome of $R R S^{W T}$ versus $R R S^{S C R}$ adult flies, in response to both gram positive and gram negative infection, led us to suggest that SUMOylation of RRS could modulate the host-defense response in Drosophila.

\section{RESULTS}

\section{The Multi-Aminoacyl tRNA Synthetase Complex Complex Is a Target for SUMO Machinery}

Proteomics studies in a host of organisms suggest that members of the MARS Complex are SUMOylation targets (Figure 1G and Supplementary Figure 1; Panse et al., 2004; Tatham et al., 2011; Handu et al., 2015), including studies in Drosophila (Handu et al., 2015; Pirone et al., 2017). Handu et al. (2015) specifically enriched proteins that changed their SUMOylation status in response to a broad activation of immune pathways, with ARSs being significant targets. As a first step to validate the targets, we cloned members of the Drosophila MARS complex (Lu et al., 2015) into bacterial expression vectors and screened their ability to be SUMOylated in an in-bacto system (Nie et al., 2009), which uses Drosophila enzymes expressed in bacteria for SUMO conjugation. We find that five ARSs; EPRS, RRS, KRS, DRS, and one AIMP (AIMP1) were modified by SUMO (Figures 1A-F). MRS and LRS could not be expressed while IRS was expressed and not SUMO conjugated. The SUMOylation status for QRS, AIMP2 and 3 was inconclusive due to low protein expression and high background in western blots. Of these we choose RRS as a target to characterize, it being an understudied target showing robust SUMOylation. Prediction of SUMO conjugation sites (Beauclair et al., 2015) in the RRS sequence suggests that RRS has a strong consensus SUMO conjugation motif at K383. Our experimental data suggested that RRS can show upto two SUMO conjugates (Figures 1H,I) and multiple rounds of mutagenesis followed by in-bacto SUMOylation led to the finding that a mutant RRS ${ }^{K 147 R, K 383 R}$ is SUMO conjugation resistant $\left(\mathrm{RRS}^{S C R}\right)$ (Figure 1I). RRS is part of subcomplex-II (Figure 1G) in the MARS complex, associating intimately with QRS and AIMP1. Analysis of the crystal structure of sub-complex-II suggests that the equivalent amino acids in the human structure [Figure 1J, 4R3Z, (Fu et al., 2014)] are not part of the protein:protein interface with either QRS or AIMP1. We generated a structural model (Supplementary Model S1) of RRS using the automated SWISS-MODEL server (Waterhouse et al., 2018), using the 4Q2T PDB structure (Kim et al., 2014a) as a homology model and mapped the two conjugation sites onto the fly model (Figure 1K). K147 is part of a low scoring SUMO target motif $(\mathrm{LKGH})$, at the end of a predicted helix, in a region that is not conserved (Figure 1L). K383 is part of a high scoring SUMO consensus motif (VKSD), in a conserved loop near the Arginine bound active site. The nearest residue which interacts with the bound Arg is F388.

\section{Generation of a Arginyl tRNA Synthetase Loss of Function Line Using CRISPR Cas9 Genome Editing}

The UAS-Gal4 system is an ideal system to express RRS ${ }^{W T}$ and RRS ${ }^{S C R}$ in a RRS loss of function (lof) background. Since such a lof line is not available, as a first step we used CRISPR Cas9 genome editing to generate the same. A transgenic dualguide RNA line (UAS-RRS $S^{d u a l-g R N A}$ ) was created (See section "Materials and Methods") to express $g R N A$ that would recognize the $5^{\prime}$ UTR and $3^{\prime}$ end of the coding region of the RRS gene (inverted red triangles, Figure 2A), near the translation start and stop sites. Our goal was to remove a major portion of the coding region to create a $\triangle R R S$ animal. The UAS-RRSdual-gRNA line was crossed to a nos-Cas 9 animal (Figure 2B) and sixty lines stabilized by balancing the putative lof's over an X chromosome balancer, FM7 $i$ where the balancer chromosome expresses GFP. Of these lines, seven were male lethal, which was indicative of a successful excision of the RRS locus, since the absence of the RRS on the $\mathrm{X}$ chromosome would lead to lethality. Single fly genomic PCRs were conducted on these lines, but the genomic PCR products did not show the expected $2.1 \mathrm{~kb}$ deletion that would be a consequence of removal of the RRS genomic region. To probe the observed male lethality, we sequenced the genomic region of two lines $6 \mathrm{~B} 1$ and $18 \mathrm{~B} 1$. To our surprise, we found that even though the coding region was not deleted, the gRNA activity caused changes to the sequence of the wildtype genome in the sites targeted by both gRNA (Figures 2C,D), and these modifications presumably led to the generation of variant allele(s). $R R S^{6 B 1}$ has a 13 bp deletion in the $5^{\prime} \mathrm{UTR}$ region (Figures 2C,D and Supplementary Figure 2A), while in the case 
A

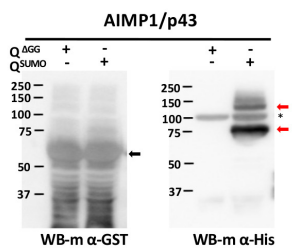

C

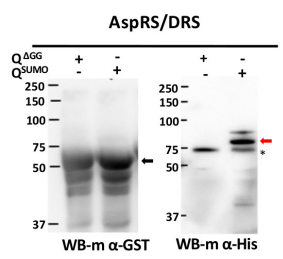

E

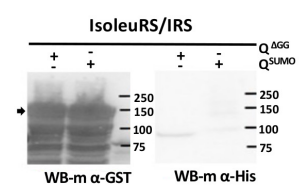

B

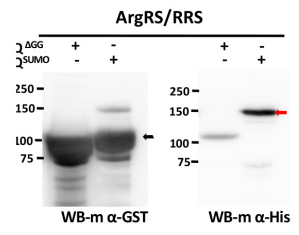

D

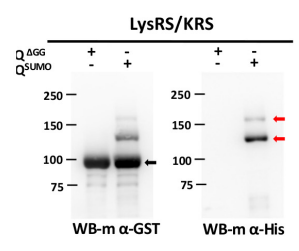

F

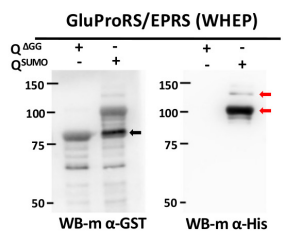

H

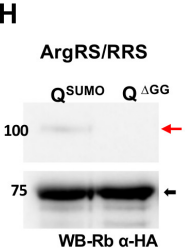

J

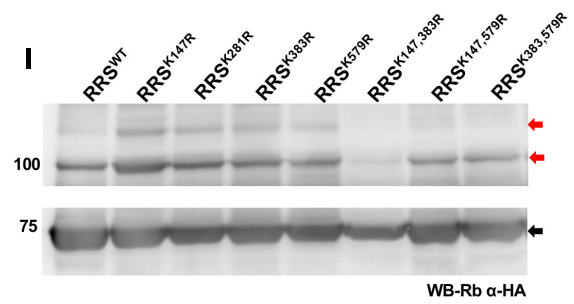

K
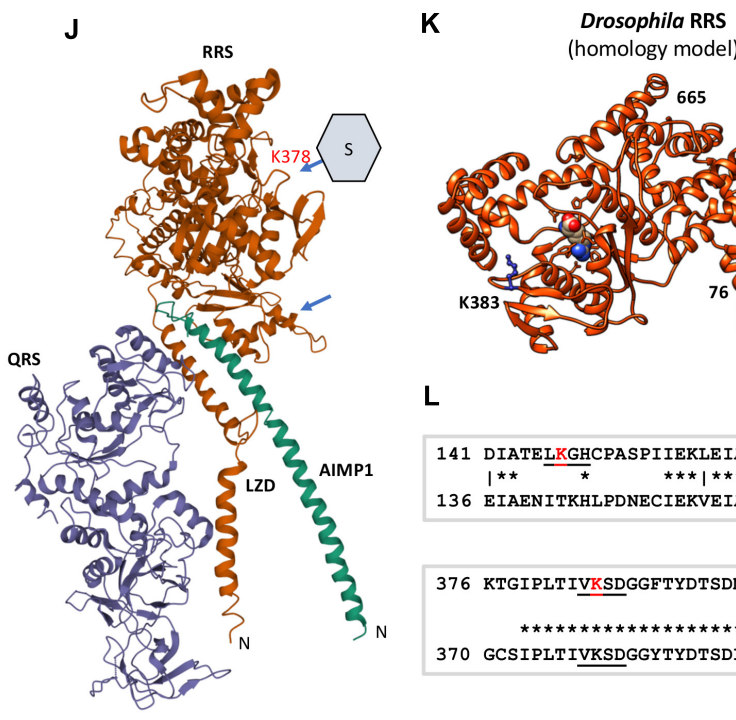

(homology model)

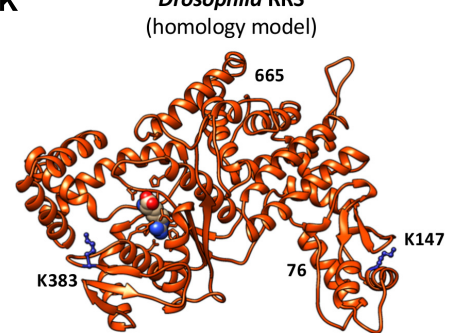

L

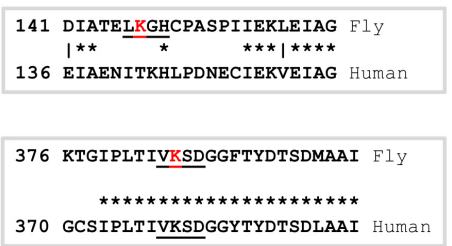

G

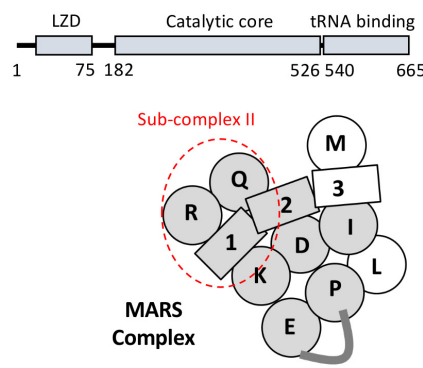
ArgRS/RRS 


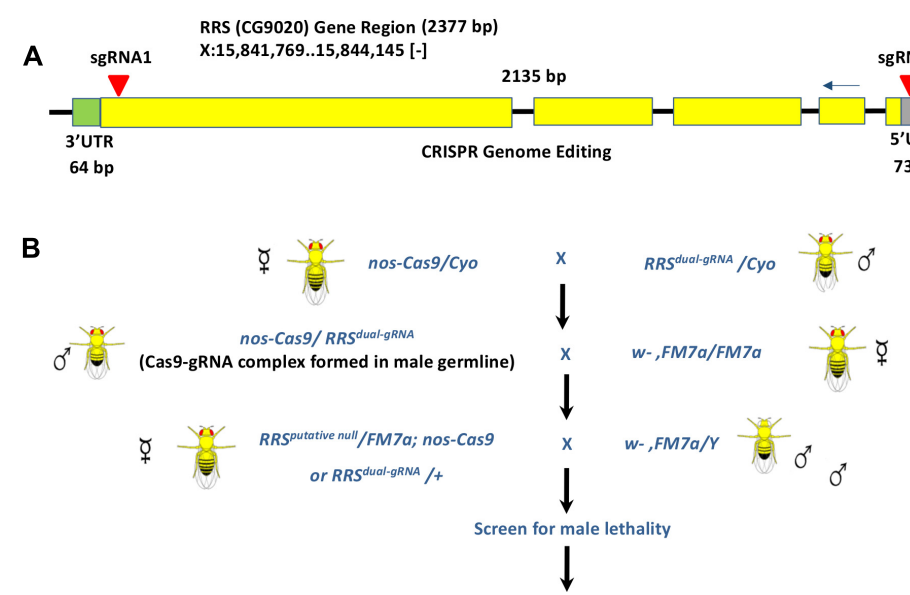

Single Fly genomic PCR (Lines 3B5, 5B4, 6B1, 12B1, 18B1, 18B7, 19B12)

\section{C}

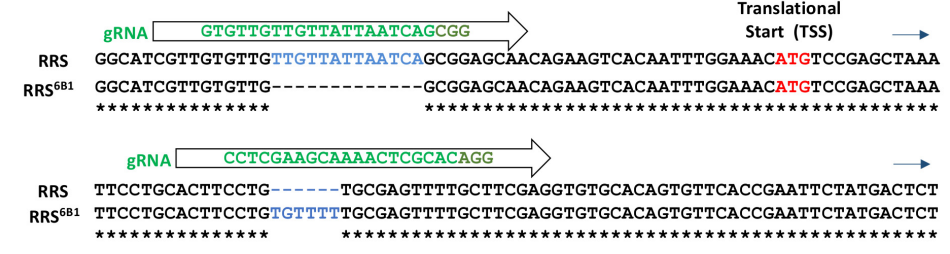

D

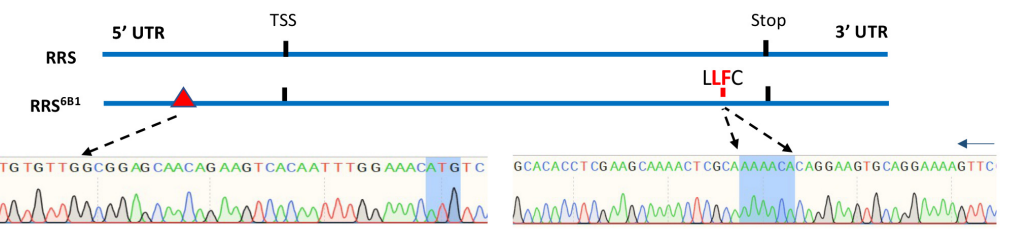

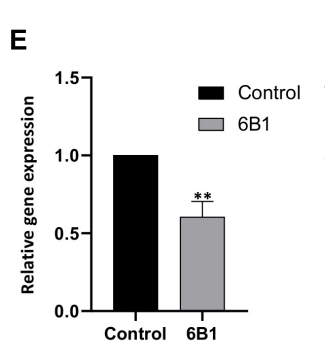

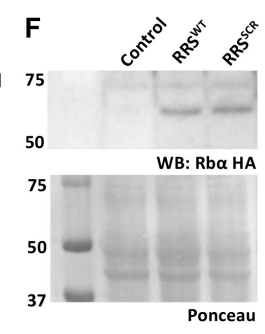

G

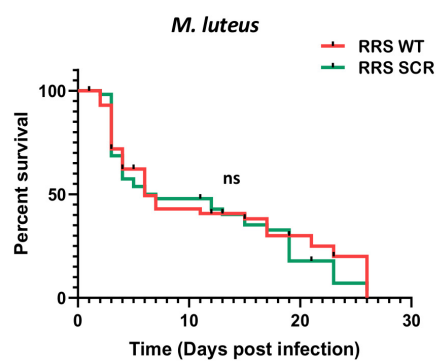

Ecc15

H

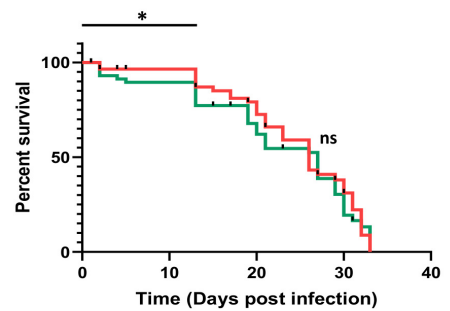

FIGURE 2 | A RRS loss of function line generated using CRISPR Cas9 genome editing. (A) Design of the dual guide-RNA for excision of the RRS locus. The RRS gene is located on the $\mathrm{X}$ chromosome. It has five exons, which code for a single annotated transcript that spans 2,377 bp. Two gRNAs (inverted red triangles) were designed in the $5^{\prime}$ UTR and $3^{\prime}$ end of the coding region. Our goal was to excise most of the coding region and generate a RRS loss of function line. (B) Excision of the RRS locus to generate a RRS-Iof line. A UAS-RRSdual-gRNA line was generated (see section "Materials and Methods") and crossed to nos-Cas9 animals. Sixty lines were balanced over a first chromosome balancer and screened for male lethality. None of the seven male lethals had the expected deletion in the RRS locus, based on PCR. (C) $R R S^{6 B 1}$ lines generated by disruption of the $5^{\prime} \mathrm{UTR}$. Genomic DNA Sequencing of the $6 \mathrm{~B} 1$ line in the RRS genomic region reveals deletions/insertions in the RRS locus, at the gRNA binding site(s). A 13 bp deletion is seen in the $5^{\prime} \mathrm{UTR}$ and a 6 bp insertion near the $3^{\prime}$ end of the coding region. (D) Schematic of the mutations in the $R R S^{6 B 1}$ line. Schematic showing the deletions near the translation start site and insertion in the coding region near the translational stop site. DNA sequences for each perturbation are also shown. (E) RRS ${ }^{6 B 1}$ line shows lower transcript levels of RRS as compared to wildtype. RRS $S^{6 B 1}$ shows $40 \%$ reduction in transcript levels of RRS as compared to wildtype Control. Values on the Y-axis depict the fold change normalized to the house-keeping gene rp49. Values shown are Mean \pm SEM. $N=3, n$ (larvae) $=25$. Statistical analysis by Unpaired $t$-test. ${ }^{*} p<0.05$ and ${ }^{* *} p<0.01$. (F) Rescue of $R R S^{6 B 1}$ by ectopic expression of RRS using UAS-Gal4 system. Both RRSWT and RRSSCR lines show approximately equal expression of RRS, when probed using an anti-HA antibody. Ponceau staining on the same blot us used to show equal loading. (G,H) Survival plots for RRSWT and RRSSCR upon M. luteus and ECc15 infection. Log rank (Mantel cox) survival plot using Kalpan-Meier and Gehan-Wilcoxon tests, suggests that RRS ${ }^{W T}$ and $R R S^{S C R}$ do not show a significant difference in lifespan post infection with $M$. luteus. However, post ECC15 infection $R R S^{S C R}$ shows a significant $\left({ }^{*} p<0.05\right)$ decrease in survival as compared to $R R S^{W T}$ in the initial stages $(0-15$ days).

fluorescence, show $40 \%$ reduction in $R R S$ transcripts as compared to $w t$ (Figure 2E), with similar results for $R R S^{18 B 1}$ (data not shown). We believe that maternal RNA still perdures at this stage, and reduces as the animals transit to the 2nd Instar. The transcript levels measured are thus a sum of maternal and zygotic RNA.

Sequence changes in the coding region were also seen in both lines (Supplementary Figures 2A-C). For $R R S^{6 B 1}$, a 6 bp insert would lead to incorporation of a Leu and Phe (Figures 2C,D) (Supplementary Figure 2A) in positions 604 and 605, within the RRS sequence; For $R R S^{18 B 1}$, the sequence corresponding to the
C-terminal domain could not be elucidated in spite of multiple sequencing attempts (Supplementary Figure 2C). For $R R S^{6 B 1}$, the insertion may perturb the structure of the C-terminal tRNA binding domain. One possible scenario is the disruption of the predicted (Craig and Dombkowski, 2013) C515:C604 disulfide bond (Supplementary Figure 2B), in the Drosophila structural model (Suppl. Model S1), which could lead to significant destabilization of RRS ${ }^{6 B 1}$ and lower its functionality. The $R R S^{6 B 1}$ line with defined mutations in the $5^{\prime}$ UTR and coding region, and with homozygotes dying in the 1 st to 2 nd Instar transition was used for all further experiments. The $6 \mathrm{~B} 1$ line is in all 
probability a hypomorphic, lof allele of RRS. The $R R S^{6 B 1} /+$ and $R R S^{18 B 1} /+$ lines are haplo-sufficient, showing normal lifespan at 25 and $29^{\circ} \mathrm{C}$ (Supplementary Figure 2D) and do not show any embryonic or larval lethality.

\section{Generation of a Transgenic RRS ${ }^{S C R}$ Line}

The successful generation of the lof $R R S^{6 B 1}$ line meant that the UAS-Gal4 system could be used to rescue the larval lethality. For this, RRS-WT and RRS-SCR sequences were cloned into a UAS vector (see section "Materials and Methods") and UAS-RRSWT and $U A S-R R S^{S C R}$ lines were created on the IIIrd chromosome. Actin-Gal4; UAS-RRS ${ }^{W T}$ and Actin-Gal4; UAS-RRS $S^{S C R}$ lines were balanced and crossed to $R R S^{6 B 1} / F M 7 i$ females. Both these lines could rescue the lethality of the $\triangle R R S$ male in the F1 generation, with the lines of the genotype, $R R S^{6 B 1}$; Actin-Gal4; UAS-RRS ${ }^{W T}$ (referred to as $R R S^{W T}$ ) and $R R S^{6 B 1}$; Actin-Gal4; UAS-RRS $S^{S C R}$ (referred to as $R R S^{S C R}$ ) being used for further experiments. Similar rescue was seen when a Ubiquitin-Gal4 was used instead of Actin-Gal4. Both the "rescued" lines were homozygous viable, had a normal lifespan, suggesting that the SCR allele was functionally equivalent to the WT in terms of its canonical function. Western blots of adult males, rescued by expression of $U A S-R R S^{S C R}$, showed equal expression of RRS, when compared to $U A S-R R S^{W T}$ (Figure $2 \mathbf{F}$ ).

Drosophila reacts to immune challenge under laboratory conditions with a characteristic transcriptional upregulation and downregulation of defense genes. Infection with gram positive Micrococcus luteus ( $M$. luteus) and gram negative Erwinia carotovora carotovora (Ecc15) were used to trigger the host-defense response. We measured the lifespan of $R R S^{W T}$ and $R R S^{S C R}$ animals post-infection. We find that there is no significant difference in lifespan for M. luteus infections, while for $E c c 15$, there is an increase in lethality for $R R S^{S C R}$, for younger animals (1-15 day), while not for older animals (20 day) (Figures 2G,H).

\section{Transcriptomics of Immune Challenged, RRS $^{W T}$ and RRS ${ }^{S C R}$ Transgenic Animals}

In order to uncover the role of SUMO conjugation in hostdefense, we infected 7-8 day old adult flies with $M$. luteus and Ecc15 and measured transcript levels in both $R R S^{W T}$ and $R R S^{S C R}$ using quantitative $3^{\prime}$ RNA sequencing (QuantSeq; see section "Materials and Methods").

Infection with the bacteria gave a robust immune response (Figures 3A-D and Supplementary Figures 3A-D). Gene Ontology analysis of the modulated genes revealed immune responsive genes associated with Gram Positive and Gram Negative infection for both common and differentially expressed genes, as expected by Toll/NFKB and Immune Deficient (Imd)/NFאB pathway activation (De Gregorio et al., 2002; Supplementary Figures 4A,B). For $R R S^{W T}$ flies, infection by $M$. luteus led to an upregulation of 66 genes and a downregulation of 2 genes, $22 \mathrm{~h}$ post infection. As expected, targets of the Toll pathway such as drosomycin (Drs) and metchnikowin (Mtk) were upregulated (Figure $3 \mathrm{E}$ and Supplementary Table 1). For the $R R S^{S C R}$ files, 85 genes were upregulated and 7 genes were downregulated. In a similar vein, infection by Ecc15 led to 232 upregulated and 151 downregulated in $R R S^{W T}$ and 209 upregulated and 79 downregulated in the $R R S^{S C R}$ (Figure 3F and Supplementary Table 2). As expected, targets of the Imd pathway were strongly modulated. In order to examine the extent of overlap among upregulated and downregulated genes between different data sets, Venn diagrams were drawn (Figures 3G,H). A majority of the genes were uniquely expressed among the data sets. Uniquely differentially expressed genes are listed in Supplementary Table 3. Common genes between $R R S^{W T}$ and $R R S^{S C R}$ for each infection category were used for further analysis. At basal level, before infection, $R R S^{W T}$ and $R R S^{S C R}$ showed minor differences in their transcriptome (Supplementary Tables 4, 5).

\section{Modulation of the Immune Transcriptome in $R R S^{S C R}$ Transgenics}

Next, we compared the change in immune transcriptome for $R R S^{S C R}$ with reference to $R R S^{W T}$ (Figures $4 \mathbf{A}, \mathbf{B}$ and Supplementary Figures 5, 6). In case of M. luteus infection, a total of 22 immune responsive genes including AMPs, Bomanins, Serine hydrolases and genes involved in ROS production were significantly differentially up-regulated (Supplementary Table 1 and Supplementary Figures 5A, 6A) in $R R S^{S C R}$. Both Drosomycin (Drs) and Bomanin Bicipital 1(BomBc1) are upregulated 5-6 fold in $R R S^{S C R}$, while other AMP genes (Figure 4A) were not strongly or significantly upregulated.

In case of Ecc15 infection, the trends were stronger. A total of 28 genes showed enhanced upregulation and 13 genes showed enhanced repression in $R R S^{S C R}$. Genes involved in metabolism, such as hydrolases, esterases, non-coding RNA and AMP genes were modulated. Amongst the strongly expressed genes (Figure 4B, Supplementary Table 2, and Supplementary Figures 5B, 6B) were immune responsive genes involved in gram-negative bacterial recognition (PGRP-LB, PGRP-LF, and PGRP-SD), and melanization (Hayan, Pale, and Punch). Genes involved in oxido-reductase pathways like Sodh 1 and Larval serum proteins $L s p 1, L s p 2$ were repressed. For the AMP genes (Figure 4B), CecA1 and $A t t D$ were upregulated 2-4 fold, while $A t t C, A t t A$, and $D p t B$ downregulated 3-9 fold. We also looked at the transcriptional changes in the genes of the MARS complex. For both M. luteus and for Ecc15, the transcriptional changes on infection were minimal, with none of the transcript levels crossing our cut-off of significance, 0.55 $\log _{2}(\mathrm{FC})$ (Figures 4C,D).

Next, we validated the QuantSeq data by qRT-PCR for a few targets at time points ranging from $0-48 \mathrm{~h}$. For M. luteus, $R R S$ levels did not change significantly from 0-48 h (Figure 4E), while Drs levels, though significant, showed similar trends over $48 \mathrm{~h}$ (Figure 4F). Irc levels were distinctly higher in $R R S^{S C R}$ animals at later time points (Figure 4G). For Ecc15, RRS transcript levels were different, showing fivefold decrease in $R R S^{S C R}$ animals, for the time-points 1.5 and $6 \mathrm{~h}$ (Figure $4 \mathrm{H}$ ). $D p t B$ and $A t t D$ transcripts are significantly lower in the case of $R R S^{S C R}$ animals (Figures 4I,J). 


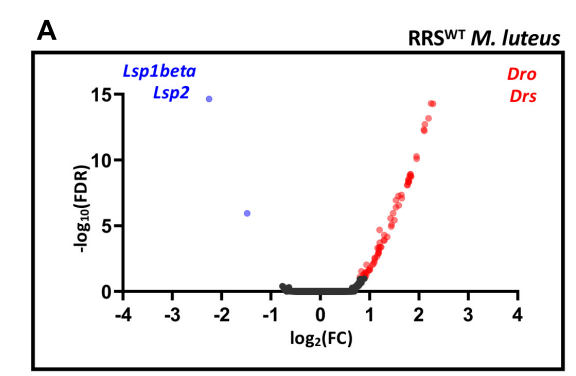

C C RRSWT Ecc15

B

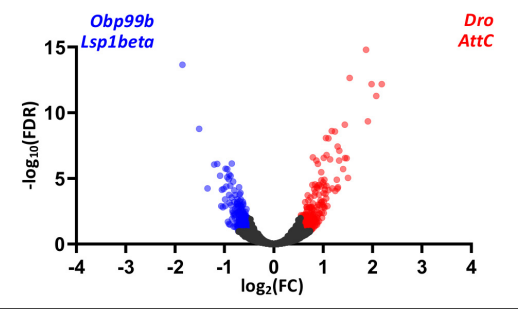

G
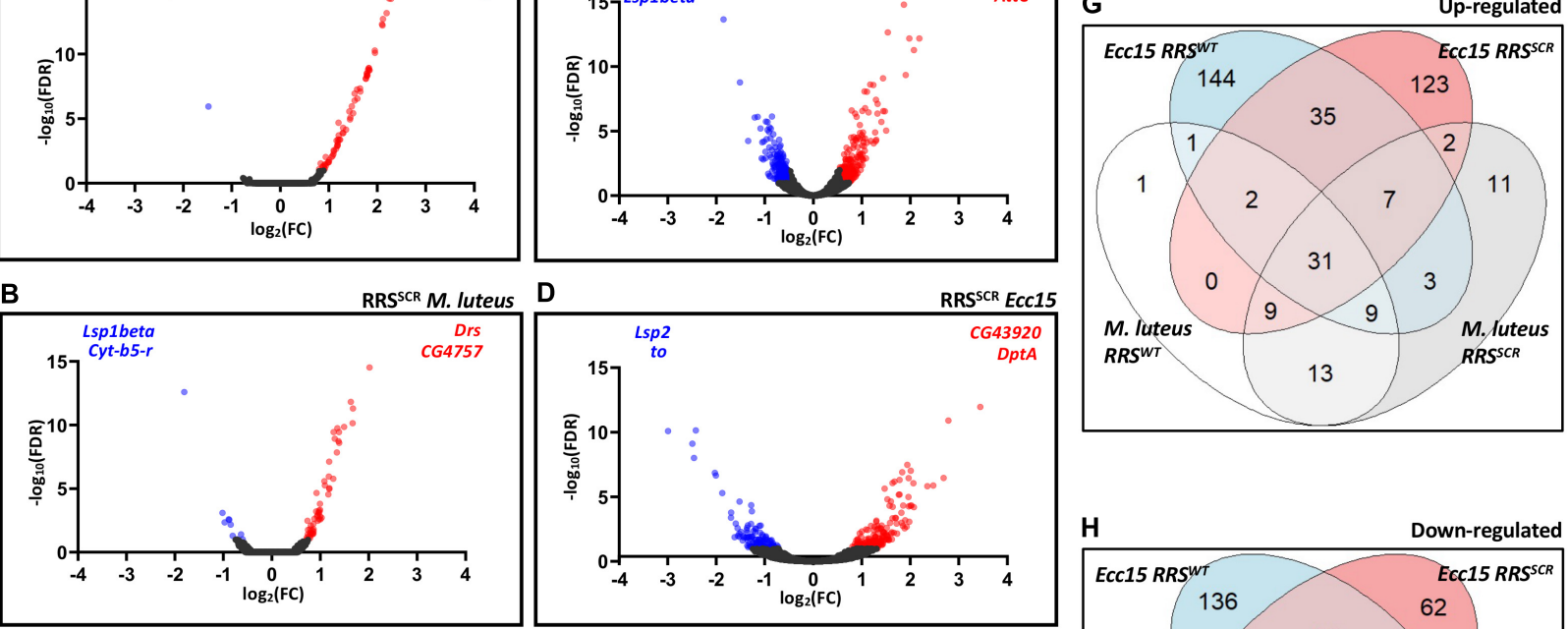

D
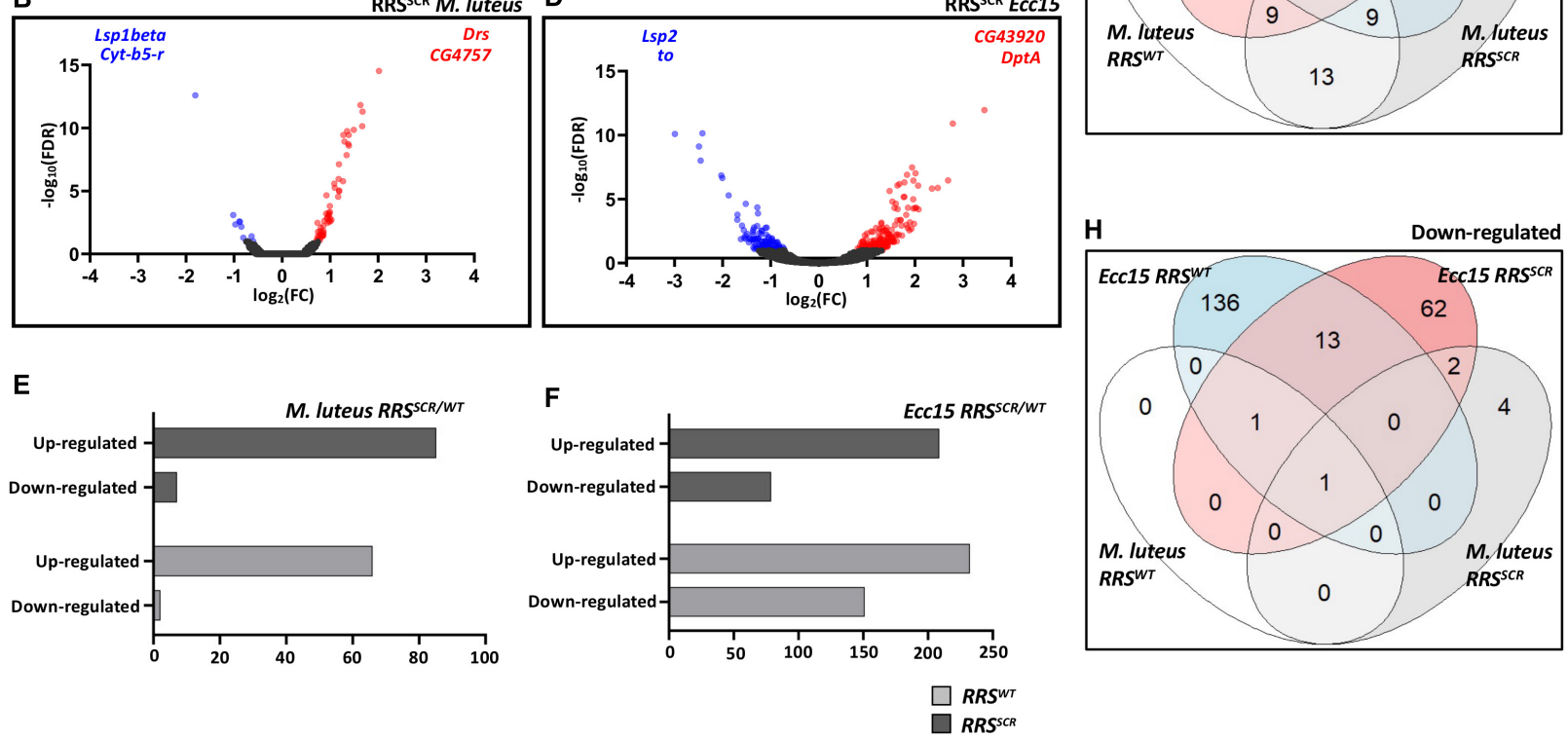

FIGURE 3 $\mid R R S^{W T}$ and $R R S^{S C R}$ show a robust immune response to bacterial infection. (A-D) Volcano plot(S) for The differentially expressed genes. log $($ FC) for each gene is plotted against its - $\log _{10}(F D R)$ value to display differentially expressed genes upon infection as compared to the baseline. Red and blue dots represent the genes which are significantly differentially expressed with $\log _{2}$ (FC) of $>0.55$ and $<-0.55$, respectively, with $p$-value $<0.05$ [-log 10 (FDR) of $>2$ ] whereas black dots represent the genes which are uniformly expressed. Representative differentially expressed genes are mentioned on the right (upregulated) and left hand (downregulated) corner of each plot. The time point for M. luteus infection is $22 \mathrm{~h}$ and for Ecc15, it is $12 \mathrm{~h}$. (E,F) Total Number of transcripts upregulated and downregulated in response to infection. Genes modulated by infection by M. luteus (E) and Ecc15 (F), for both RRSWT and RRSSCR . (G,H) Differential expression of genes. Venn diagram showing sub-division of upregulated $\mathbf{( G )}$ and downregulated $\mathbf{( H )}$ genes for experiments conducted, as defined earlier.

\section{DISCUSSION}

The MARS Complex has been implicated as a sensor and regulator of the immune response (Guo and Schimmel, 2013; Kim et al., 2014b; Arif et al., 2018; Nie et al., 2019). Mutations and mis-regulation of MARS function can lead to immune disease (Lee et al., 2018; Nie et al., 2019). In the best studied mechanistic example, in response to infection and release of IFN- $\gamma$, EPRS dissociates from the MARS Complex (Sampath et al., 2004). The dissociation is triggered by phosphorylation of the WHEP domain. EPRS now associates with L13a, NSAP1 and GAPDH to form a "GAIT complex" which can now bind to a GAIT RNA element. The GAIT-RNA element (interferon-gamma-activated inhibitor of translation) (Sampath et al., 2003; Marquez-Jurado et al., 2015) is present in UTRs of mRNA transcripts and binding leads to a block of translation of the transcript.

Roles for RRS in the immune response are unknown. In terms of disease, mutations in RRS have been implicated in neuronal hypomyelination with severe spasticity and nystagmus (Antonellis and Green, 2008; Wolf et al., 2014). Autoantibodies against ARSs were found in anti-synthetase syndrome (ASSD), suggesting that ARSs are likely to be involved in the development and progression of autoimmune disease. In Drosophila, RRS is not studied in any physiological context.

How then does RRS modulate transcription of defense genes? In mammals, the MARS complex itself is believed to be a cytoplasmic complex, though a few studies suggest nuclear localization (Wolfe et al., 2003; Cui et al., 2020). RRS could be available in at least three species, one as a free, unbound entity, second as a complex with AIMP1 and QRS and finally as part of the MARS Complex (Figure 4L). Deletion of the RRS LZD leads to its dissociation of the MARS complex, but this does not affect charging (Cui et al., 2020). Interestingly, the nuclear fraction of MARS decreases when cells contain RRS ( $\triangle \mathrm{LZD})$. In its dissociated state, RRS's canonical functions are unaffected, but developmental genes 


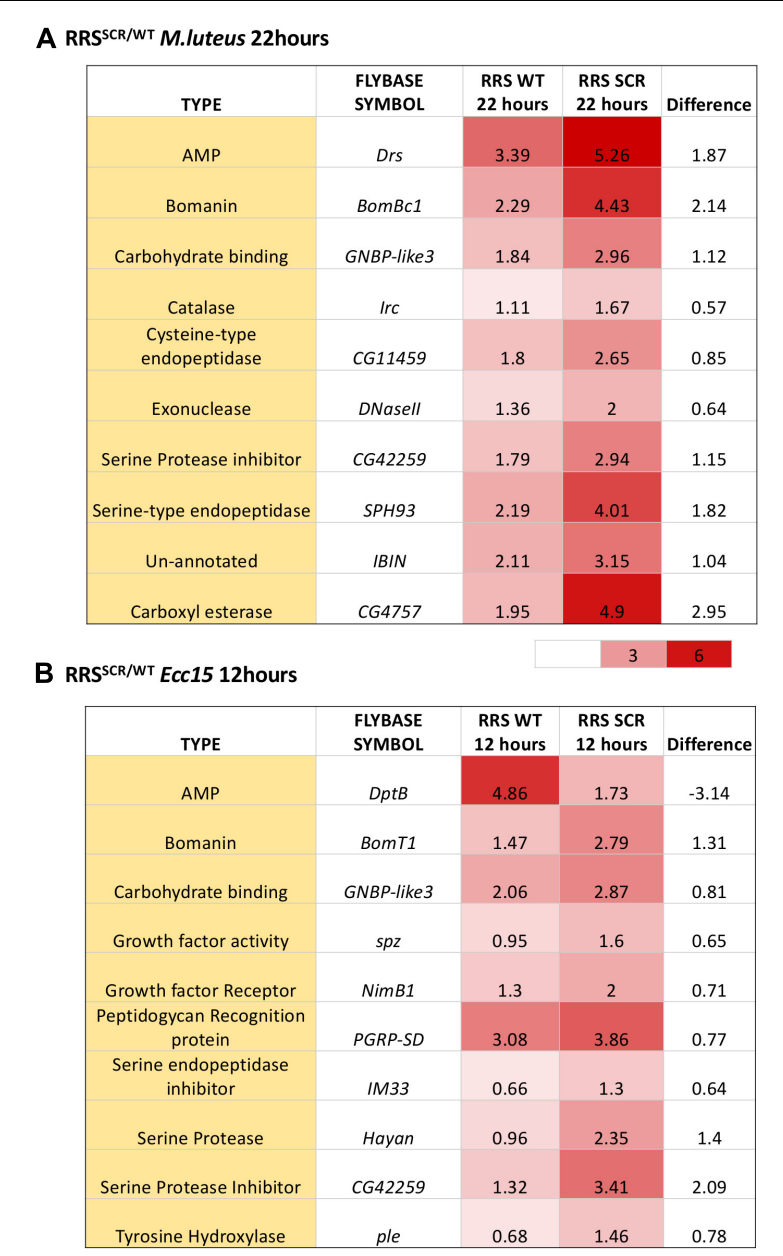

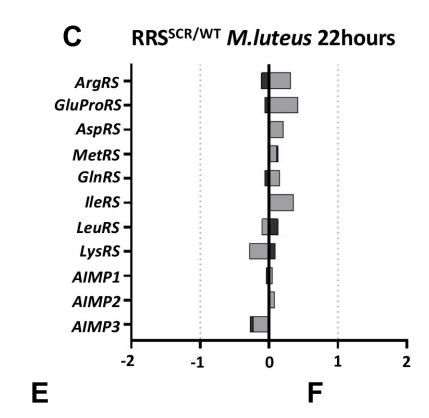
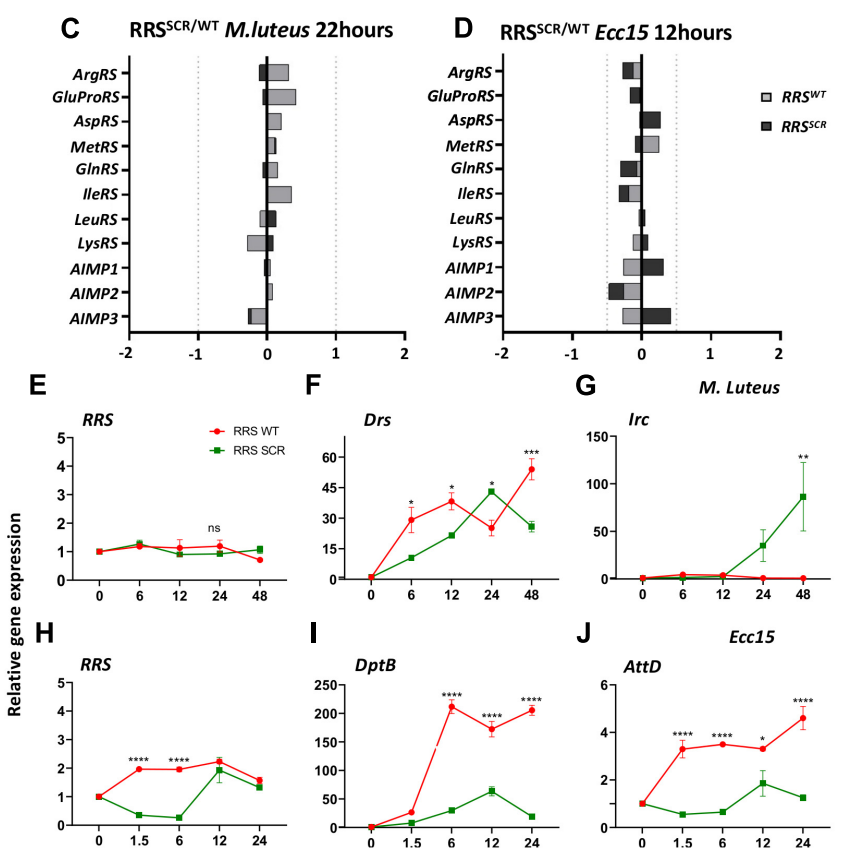

Time lapsed post infection (hours)
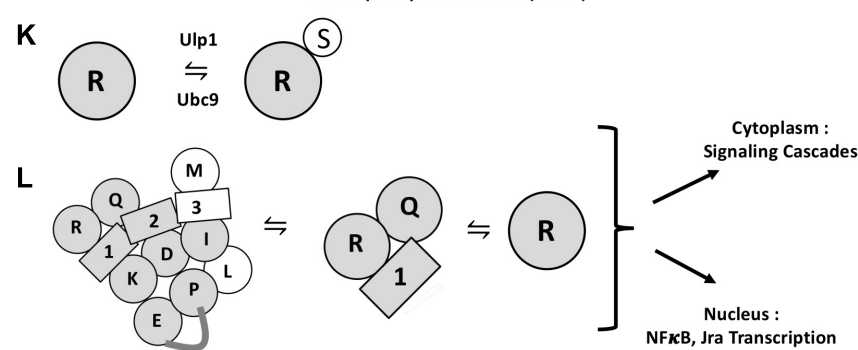

FIGURE 4 | RRS ${ }^{W T}$ and RRS ${ }^{S C R}$ show differential expression of immune target genes. (A,B). Tabulation of differentially expressed genes. Representative genes with differential expression for $R R S^{S C R}$ for M. luteus (A) and $E_{C C 15}$ (B) infections. For M. luteus, there is moderate upregulation for most genes, while for $E_{C C 15}$, metabolic genes are both up and down regulated. (C,D) Transcriptome changes for MARS Complex genes. On infection by M. luteus (C) and Ecc15 (D), the changes in transcript levels for genes that code for proteins in the MARS complex is well below the significance cut-off of $0.5 \log _{2}(\mathrm{FC})$. (E-G) Expression of RRS, Toll pathway target gene Drosomycin and Immune regulated catalase (Irc) in RRSWT and RRS SCR upon M. luteus infection across $0-48 \mathrm{~h}$. Values on the Y-axis depict the fold change normalized to the house-keeping gene rp49. Values shown are Mean + SEM. $N=3, n=5$. Statistical analysis by two-way ANOVA followed by Tukey's multiple comparison test. ${ }^{\star} p<0.05,{ }^{\star \star} p<0.01,{ }^{\star * \star} p<0.001$, and ${ }^{* \star \star *} p<0.0001$. (H-J) Expression of RRS, Imd pathway target genes Diptericin B and Attacin D in RRS WT and RRS ${ }^{S C R}$ upon ECC15 infection across $0-24 \mathrm{~h}$. Values on the Y-axis depict the fold change normalized to the house-keeping gene rp49. Values shown are Mean \pm SEM. $N=3, n=5$. Statistical analysis by two-way ANOVA followed by Tukey's multiple comparison test. ${ }^{*} p<0.05,{ }^{* *} p<0.01$, ${ }^{* \star *} p<0.001$, and ${ }^{\star \star \star *} p<0.0001$. (K) SUMO Conjugation of RRS. RRS is a target for the cellular SUMO conjugation machinery. A small fraction of RRS is SUMO conjugated and is in equilibrium with non-conjugated RRS. (L) Model for immune regulation by RRS. RRS may influence the immune response either as part of the MARS complex, or as part of the AIMP1:RRS:QRS complex or as free RRS. In a SUMO-conjugated state, RRS may influence signaling cascades, interacting with partners containing SIM sites and modify their function. These influences can be either cytoplasmic or nuclear.

such as homeobox and forkhead box genes are modulated (Cui et al., 2020).

Each of the RRS species could exist in a SUMO conjugated or unconjugated state (Figure $4 \mathrm{~K}$ ). These species can ultimately regulate gene expression either by influencing signaling pathways in the cytoplasm or by affecting the transcription of the nuclear localized NFאBs. SUMOylation could affect the stability or interaction with other proteins. RRS lacks a nuclear localization signal (NLS), as does Drosophila SUMO. Transport to the nucleus would require RRS to be part of a complex that includes a NLS, for example the AIMP1:RRS:QRS complex, as AIMP1 may travel to the nucleus (Lee et al., 2008; Park et al., 2010). AIMP1 in mammals is a precursor for EMAPII which can trigger an inflammatory response (Lee et al., 2019) and a similar mechanism may exist in flies. Other possible mechanisms include modulation of NFKB (Ko et al., 2001) by regulation of secretion of AIMP1 or by regulation of Jun signaling (Park et al., 2002), which in turn can regulate the immune response.

In Summary, RRS is SUMO conjugated and SUMOylation appears to modulate, indirectly the transcriptional host-defense response. The mechanisms underlying these phenomena are currently unknown. 


\section{MATERIALS AND METHODS}

\section{SUMO Conjugation Assay}

SUMOylation of constituents of the MARS complex was tested by expressing the target/substrate protein simultaneously with the Drosophila SUMO cycle components based on a published protocol (Nie et al., 2019). Target proteins from the Drosophila Gold cDNA collection, procured from the Drosophila Genome Resource Center (DGRC), Bloomington, Indiana were subcloned into $p G E X-4 T 1$ (Promega) and $p E T-45 b$, and subsequently sequenced for validation. For visualization of SUMO conjugation, bacterial lysates were affinity purified using Glutathione-Agarose beads (Invitrogen) or Ni NTA-Agarose beads (Qiagen), run on an SDS-PAGE gel and monitored using mouse anti-GST antibody (sc53909, 1:5,000; Santa-Cruz-Biotechnology), Rabbit anti-HA antibody (DW2, 1:3,000; Millipore) and mouse anti-6XHis antibody (H1029, 1:1,000; SIGMA) using Western blotting. The SUMO conjugated forms appear as bands of a higher molecular weight.

\section{SUMO-Binding-Motif and SIM-Motif Prediction}

Putative SUMO acceptor lysines and SIM-motifs of all the MARS complex components of Drosophila were predicted in silico, using Joined Advanced SUMOylation and Sim motif Analyzer (JASSA) tool with a threshold cut-off criteria set at "high" (Beauclair et al., 2015).

\section{Identification of Evolutionarily Conserved SUMO Target Lysine Residues in silico}

FASTA sequences of RRS for model organisms belonging to different eukaryotic groups were procured from the Uniprot protein database. Multiple sequence alignment (MSA) was done on the basis of homology extension using PSI-COFFEE (Chang et al., 2012). SUMO acceptor lysines were compared across different representative organisms, post-alignment.

\section{Homology Model for Drosophila Arginyl tRNA Synthetase}

The automated SWISS-MODEL server (Waterhouse et al., 2018) was used to generate a structural models (RRS $\left.{ }^{W T}, \mathrm{RRS}^{6 B 1}\right)$ using default parameters. The human 4Q2T PDB structure (Kim et al., $2014 \mathrm{a}$ ), solved at a resolution of $2.4 \AA$, containing a bound Arginine at the active site was used as a template.

\section{Generation of Arginyl tRNA Synthetase Loss of Function Lines Using CRISPR Cas9 Technology}

CRISPR Cas9 technology was employed to generate RRS loss of function fly lines. Single guide (sg)-RNAs targeting the RRS coding region in the $5^{\prime} \mathrm{UTR}$ and Exon-5 were designed using CRISPR Optimal Target Finder [COTF; (Gratz et al., 2014)], a web tool for identifying CRISPR target sites and evaluating their specificity. The RRS gene region was sequenced prior to the experiment to designing the gRNAs to account for SNPs at the sgRNA target sites. The sgRNAs were cloned into the pU6BbsI-chiRNA (Addgene \# 45946) plasmid, which was then docked into $y^{1} v^{1}$; P\{CaryP\}attP40 Drosophila line (BDSC 36304), by transgenic injections, at the NCBS-CCAMP transgenic facility, Bangalore, India. The transgenic dual sgRNA line was crossed to nanos-Cas9 (BDSC 54591) line. The founder male progenies obtained were crossed to $w$-; FM7a balancer females wherein the Cas9-sgRNA complex is formed in the germline. In the next generation, three heterozygous female progenies from each cross (60 lines, each labeled A, B, and C) were maintained as a separate line over a FM7a balancer. Since the genomic RRS is located on the $\mathrm{X}$ chromosome, putative $R R S$ lof lines were screened for male lethality. Lines showing male lethality were chosen for PCRbased confirmation of the deletion. Single fly genomic PCR for the extended gene region of $R R S$ was performed on heterozygous females and the mutations were confirmed through sequencing.

\section{pUASp-AttB Fly Lines/Strains}

Arginyl tRNA Synthetase-WT and RRS-SCR(K147R,K383R) were sub-cloned into $p U A S p$-attP2 using a homology based recombination technique, a modification of the SLiCE protocol (Zhang et al., 2014). These were injected into AttB lines for generation of transgenic fly lines. Fly lines were balanced with ubiquitously expressing Gal4s (Actin-Gal4/Ubiquitin-Gal4) of the following genotype Actin-Gal4/ + ; UAS-RRS-WT/ + , ActinGal4/ + ; UAS-RRS-SCR(2MT), Ubiquitin-gal4/ + ; UAS-RRS$W T /+$ and Ubiquitin-Gal4/ + :UAS SCR(2MT). All experiments were carried out with the Actin-Gal4 line.

\section{Culturing and Processing Bacteria for Infections}

Micrococcus luteus and Ecc15 were plated on Luria-Bertani (LB) agar plates and grown in LB broth under antibiotic selection. Bacteria were collected from the plate or pelleted and resuspended in $1 \mathrm{X}$ PBS to make a concentrated solution.

\section{Fly Infections}

Six to eight day-old males were collected and placed at $29^{\circ} \mathrm{C}$ for $48 \mathrm{~h}$, to acclimatize the flies to infection temperature. To cause septic injury, flies were pricked in the thorax with a needle dipped in the concentrated solution of bacteria. To activate the Tollpathway and Imd-pathway, flies were infected with $M$. luteus and Ecc15, respectively, at an Optical density of 100, measured at $600 \mathrm{~nm}$. To measure gene expression levels, infected flies and non-infected controls were incubated at $29^{\circ} \mathrm{C}$ for the time required, after which they were collected by snap freezing them in liquid nitrogen and stored at $-80^{\circ} \mathrm{C}$ until RNA extraction. Infectivity assays were done in three biological replicates, ten flies per replicate. For survival experiments, flies were pricked in the same way as for the gene expression measurements.

\section{Total RNA Extraction cDNA Library Construction and Sequencing}

Total RNA was extracted from adult flies with following genotypes $R R S^{6 B 1} / Y$; Actin Gal4/ + ; UAS-RRS WT/ + and $R R S^{6 B 1} / Y$; Actin Gal4/ + ; UAS-RRS SCR/ +, 10 days post eclosion, 
in triplicates using RNeasy Plus Universal Kits (Qiagen; Part \#74104) under control and infected conditions, according to manufacturer's instructions and RNA integrity was assessed. 3' mRNA specific libraries were amplified using QuantSeq 3' mRNA-Seq Library Prep Kit FWD using the manufacturer's instructions. Quality assessment for the cDNA libraries was done using Bioanalyzer 2100 (Agilent Technologies). Single end $75 \mathrm{bp}$ sequencing of the pooled libraries were performed on the Illumina NextSeq 500 platform.

\section{Demultiplexing, Adapter Trimming, Read Mapping, Counts Generation and Differential Expression Analysis}

On average 4-5 million reads were generated per sample. The raw reads were demultiplexed using bcl2fastq and the adapters were trimmed using bbduk v35.92. Sequencing quality was assessed using FastQC v0.11.5. Post quality control, the reads were mapped to the Drosophila genome (dm6) using STAR aligner v.2.5.2a (Dobin et al., 2013). Gene expression levels were measured using the counts generated by HTSeq-count $\mathrm{v}$ 0.6.0 (Anders et al., 2015). The gene expression counts were normalized for all samples together and the biological conditions were compared pairwise using DESeq2 (Love et al., 2014). The Principle Component Analysis using the " $R$ " package of the regularized log counts were used to remove outliers from the final differential expression analysis. The regularized log transformed counts of the transcripts from DESeq 2 were used to determine upregulated and downregulated genes across biological samples. Genes with $\log _{2}(\mathrm{FC})$ values $\geq 0.55, \leq-0.55$ and $-\log _{10}(\mathrm{FDR})$ values $\geq 2$ were considered for further analysis. Gene Ontology analysis was done using a subroutine in Flybase.

Custom Venn diagrams were made using the Venneuler package in $\mathrm{R}$ to show the overlap and differences between the differentially expressed gene lists. Volcano plots were made using Graphpad Prism 8.0.2 for visual identification of genes with large fold changes that are also statistically significant.

\section{Survival Analysis}

Survival assays were carried out on $R R S^{W T}$ and $R R S^{S C R}$ flies (Figures 2G,H). For each experiment flies were infected 10 days post eclosion. $\sim 40$ age-matched male flies of the desired genotype were collected, each vial containing 10 flies. Animals were flipped to a fresh vial every 5 days, with number of flies recorded per vial daily. The survival data was plotted and analyzed using the log-rank test in Prism 8.

\section{Real Time-PCR}

mRNA was extracted from 10-day old adults post infection using Qiagen RNeasy mini kit (74104). 500 ng of RNA was used for the cDNA synthesis using the High Capacity cDNA Reverse Transcriptase Kit (4368814) by Applied Biosystems. The qPCR reaction was carried out using KAPA SYBR FAST (KK4602) Sigma using Analytik Jena-qTOWER ${ }^{3}$ - Real Time PCR Thermal Cycler. The experiments were carried out in triplicates with two technical replicates each. The relative fold change for each genotype was calculated by normalizing to house-keeping gene rp49. The data was analyzed by Two-way ANOVA followed by Tukey's test for multiple comparison. The primer pairs used are listed in the Resource Table (Supplementary Figure 7).

\section{DATA AVAILABILITY STATEMENT}

The original contributions presented in the study are included in the article/Supplementary Material, further inquiries can be directed to the corresponding author/s.

\section{AUTHOR CONTRIBUTIONS}

PN and GR conceptualized the project, designed the experiments, analyzed the data, and wrote the manuscript. PN and AK performed all the experiments. GR supervised the project and acquired funding. All authors contributed to the article and approved the submitted version.

\section{FUNDING}

Genome Engineering Technology (GET) grant Department of Biotechnology_GET grant (BT/PR26095/GET/119/199/2017), Department of Biotechnology (DBT), Govt. of India. The IISER Drosophila media and Stock center was supported by National Facility for Gene Function in Health and Disease (NFGFHD) at IISER Pune, which in turn is supported by an Infrastructure grant from the DBT, Govt. of India (BT/INF/22/SP17358/2016). We would like to thank Bloomington Drosophila Stock Center (BDSC), Indiana, supported by NIH grant P40OD018537, for fly stocks; Fly facility at the National Centre for Biological Sciences (NCBS), Bangalore for embryonic injections. PN is a graduate student funded by a fellowship from University Grants Commission (UGC), Govt. of India.

\section{ACKNOWLEDGMENTS}

We would like to thank Deepti Trivedi for her input on design of the dual gRNA construct for RRS.

\section{SUPPLEMENTARY MATERIAL}

The Supplementary Material for this article can be found online at: https://www.frontiersin.org/articles/10.3389/fcell.2021. 695630/full\#supplementary-material

\section{Supplementary Figure 1 | SUMO conjugated proteins based on proteomic studies.}

Supplementary Figure 2 | (A) Multiple sequence alignment of Drosophila RRS and $R R S^{6 B 1}$ DNA sequences (B). Structural consequences of CRISPR/Cas9 mediated insertions in $R R S^{6 B 1}$ flies (C). DNA sequencing data for $R R S^{18 B 1}$ (D) RRS CRISPR variant flies are haplo-sufficient and do not show embryonic lethality.

Supplementary Figure 3 | (A-D) Expanded volcano plots for differentially expressed genes. 
Supplementary Figure 4 | Gene Ontology (GO) analysis of the significantly differentially regulated genes in RRS ${ }^{W T}$ and $R_{R S}{ }^{S C R}$ post infection M. luteus (A) ECC15 (B).

Supplementary Figure 5 | (A,B) Gene Ontology (GO) analysis of the significantly differentially regulated genes in $R R S^{S C R / W T}$ post infection.

Supplementary Figure 6 | (A,B) Gene Ontology (GO) Enrichment Analysis of the significantly differentially regulated genes in $R R S S C R / W T$ post infection.

Supplementary Figure 7 | Resource Table.

Supplementary Table 1 | XLS file with processed data on gene expression levels after $M$. luteus infection.

\section{REFERENCES}

Anders, S., Pyl, P. T., and Huber, W. (2015). HTSeq-a Python framework to work with high-throughput sequencing data. Bioinformatics 31, 166-169. doi: 10.1093/bioinformatics/btu638

Antonellis, A., and Green, E. D. (2008). The role of aminoacyl-tRNA synthetases in genetic diseases. Annu. Rev. Genomics Hum. Genet. 9, 87-107. doi: 10.1146/ annurev.genom.9.081307.164204

Arif, A., Jia, J., Halawani, D., and Fox, P. L. (2017). Experimental approaches for investigation of aminoacyl tRNA synthetase phosphorylation. Methods 113, 72-82. doi: 10.1016/j.ymeth.2016.10.004

Arif, A., Yao, P., Terenzi, F., Jia, J., Ray, P. S., and Fox, P. L. (2018). The GAIT translational control system. Wiley Interdiscip. Rev. RNA 9:e1441. doi: 10.1002/ wrna.1441

Beauclair, G., Bridier-Nahmias, A., Zagury, J. F., Saib, A., and Zamborlini, A (2015). JASSA: a comprehensive tool for prediction of SUMOylation sites and SIMs. Bioinformatics 31, 3483-3491. doi: 10.1093/bioinformatics/bt v403

Chang, J. M., Di Tommaso, P., Taly, J. F., and Notredame, C. (2012). Accurate multiple sequence alignment of transmembrane proteins with PSI-Coffee. BMC Bioinformatics 13(Suppl. 4):S1. doi: 10.1186/1471-2105-13-S4-S1

Craig, D. B., and Dombkowski, A. A. (2013). Disulfide by Design 2.0: a webbased tool for disulfide engineering in proteins. BMC Bioinformatics 14:346. doi: 10.1186/1471-2105-14-346

Cui, H., Kapur, M., Diedrich, J. K., Iii, J. R. Y., Ackerman, S. L., and Schimmel, P. (2020). Regulation of ex-translational activities is the primary function of the multi-tRNA synthetase complex. Nucleic Acids Res. 49, 3603-3616. doi: $10.1093 /$ nar/gkaa1183

Curran, J. A., and Weiss, B. (2016). What Is the Impact of mRNA 5' TL heterogeneity on translational start site selection and the mammalian cellular phenotype? Front. Genet. 7:156. doi: 10.3389/fgene.2016.0 0156

De Gregorio, E., Spellman, P. T., Tzou, P., Rubin, G. M., and Lemaitre, B. (2002). The Toll and Imd pathways are the major regulators of the immune response in Drosophila. EMBO J. 21, 2568-2579. doi: 10.1093/emboj/21.11.2568

Dobin, A., Davis, C. A., Schlesinger, F., Drenkow, J., Zaleski, C., Jha, S., et al. (2013). STAR: ultrafast universal RNA-seq aligner. Bioinformatics 29, 15-21. doi: 10.1093/bioinformatics/bts635

Fu, Y., Kim, Y., Jin, K. S., Kim, H. S., Kim, J. H., Wang, D., et al. (2014). Structure of the ArgRS-GInRS-AIMP1 complex and its implications for mammalian translation. Proc. Natl. Acad. Sci. U.S.A. 111, 15084-15089. doi: 10.1073/pnas. 1408836111

Geiss-Friedlander, R., and Melchior, F. (2007). Concepts in sumoylation: a decade on. Nat. Rev. Mol. Cell Biol. 8, 947-956. doi: 10.1038/nrm2293

Golebiowski, F., Matic, I., Tatham, M. H., Cole, C., Yin, Y., Nakamura, A., et al. (2009). System-wide changes to SUMO modifications in response to heat shock. Sci. Signal. 2:ra24. doi: 10.1126/scisignal.2000282

Gratz, S. J., Ukken, F. P., Rubinstein, C. D., Thiede, G., Donohue, L. K., Cummings, A. M., et al. (2014). Highly specific and efficient CRISPR/Cas9-catalyzed homology-directed repair in Drosophila. Genetics 196, 961-971. doi: 10.1534/ genetics.113.160713

Guo, M., and Schimmel, P. (2013). Essential nontranslational functions of tRNA synthetases. Nat. Chem. Biol. 9, 145-153. doi: 10.1038/nchembio.1158
Supplementary Table 2 |XLS file with processed data on gene expression levels after Ecc 15 infection.

Supplementary Table 3 |XLS file with processed data for uniquely expressed genes for both $M$. luteus and ECC 15, post infection.

Supplementary Table 4 | XLS file with processed data for differentially expressed genes for M. luteus, without infection (0 h).

Supplementary Table 5 |XLS file with processed data for differentially expressed genes Ecc 15, without infection (0 h).

Supplementary Model S1 | Drosophila RRS structural model generated by the automated SWISS-MODEL server, in PDB format.

Handu, M., Kaduskar, B., Ravindranathan, R., Soory, A., Giri, R., Elango, V. B., et al. (2015). SUMO-enriched proteome for Drosophila innate immune response. G3 Genes Genomes Genet. 5, 2137-2154. doi: 10.1534/g3.115.020958

Havrylenko, S., and Mirande, M. (2015). Aminoacyl-tRNA synthetase complexes in evolution. Int. J. Mol. Sci. 16, 6571-6594. doi: 10.3390/ijms16036571

Hay, R. T. (2005). SUMO: a history of modification. Mol. Cell. 18, 1-12. doi: 10.1016/j.molcel.2005.03.012

Hendriks, I. A., and Vertegaal, A. C. (2016). A comprehensive compilation of SUMO proteomics. Nat. Rev. Mol. Cell Biol. 17, 581-595. doi: 10.1038/nrm. 2016.81

Kerjan, P., Cerini, C., Semeriva, M., and Mirande, M. (1994). The multienzyme complex containing nine aminoacyl-tRNA synthetases is ubiquitous from Drosophila to mammals. Biochim. Biophys. Acta 1199, 293-297. doi: 10.1016/ 0304-4165(94)90009-4

Khan, K., Baleanu-Gogonea, C., Willard, B., Gogonea, V., and Fox, P. L. (2020). 3-Dimensional architecture of the human multi-tRNA synthetase complex. Nucleic Acids Res. 48, 8740-8754. doi: 10.1093/nar/gkaa569

Kim, H. S., Cha, S. Y., Jo, C. H., Han, A., and Hwang, K. Y. (2014a). The crystal structure of arginyl-tRNA synthetase from Homo sapiens. FEBS Lett. 588, 2328-2334. doi: 10.1016/j.febslet.2014.05.027

Kim, J. H., Han, J. M., and Kim, S. (2014b). Protein-protein interactions and multicomponent complexes of aminoacyl-tRNA synthetases. Top. Curr. Chem. 344 119-144. doi: 10.1007/128_2013_479

Ko, Y. G., Park, H., Kim, T., Lee, J. W., Park, S. G., Seol, W., et al. (2001). A cofactor of tRNA synthetase, p43, is secreted to up-regulate proinflammatory genes. J. Biol. Chem. 276, 23028-23033. doi: 10.1074/jbc.M101544200

Lee, D. D., Hochstetler, A., Murphy, C., Lowe, C. W., and Schwarz, M. A. (2019). A distinct transcriptional profile in response to endothelial monocyte activating polypeptide II is partially mediated by JAK-STAT3 in murine macrophages. Am. J. Physiol. Cell Physiol. 317, C449-C456. doi: 10.1152/ajpcell.00277.2018

Lee, E. Y., Kim, S., and Kim, M. H. (2018). Aminoacyl-tRNA synthetases, therapeutic targets for infectious diseases. Biochem. Pharmacol. 154, 424-434. doi: 10.1016/j.bcp.2018.06.009

Lee, Y. S., Han, J. M., Son, S. H., Choi, J. W., Jeon, E. J., Bae, S. C., et al. (2008). AIMP1/p43 downregulates TGF-beta signaling via stabilization of smurf2. Biochem. Biophys. Res. Commun. 371, 395-400. doi: 10.1016/j.bbrc.2008.04.099

Leppek, K., Das, R., and Barna, M. (2018). Functional 5' UTR mRNA structures in eukaryotic translation regulation and how to find them. Nat. Rev. Mol. Cell Biol. 19, 158-174. doi: 10.1038/nrm.2017.103

Love, M. I., Huber, W., and Anders, S. (2014). Moderated estimation of fold change and dispersion for RNA-seq data with DESeq2. Genome Biol. 15:550. doi: 10.1186/s13059-014-0550-8

Lu, J. M., Marygold, S. J., Gharib, W. H., and Suter, B. (2015). The aminoacyl-tRNA synthetases of Drosophila melanogaster. Fly 9, 53-61. doi: 10.1080/19336934. 2015.1101196

Marquez-Jurado, S., Nogales, A., Zuniga, S., Enjuanes, L., and Almazan, F. (2015). Identification of a gamma interferon-activated inhibitor of translation-like RNA motif at the 3' end of the transmissible gastroenteritis coronavirus genome modulating innate immune response. mBio 6:e00105. doi: 10.1128/mBio. 00105- 15

Nie, A. Z., Sun, B., Fu, Z. H., and Yu, D. S. (2019). Roles of aminoacyl-tRNA synthetases in immune regulation and immune diseases. Cell Death Dis. 10:901. doi: 10.1038/s41419-019-2145-5 
Nie, M. H., Xie, Y. M., Loo, J. A., and Courey, A. J. (2009). Genetic and proteomic evidence for roles of Drosophila SUMO in cell cycle control, ras signaling, and early pattern formation. PLoS One 4:e5905. doi: 10.1371/journal.pone.0005905

Panse, V. G., Hardeland, U., Werner, T., Kuster, B., and Hurt, E. (2004). A proteome-wide approach identifies sumoylated substrate proteins in yeast. J. Biol. Chem. 279, 41346-41351. doi: 10.1074/jbc.M407950200

Park, S. G., Choi, E. C., and Kim, S. (2010). Aminoacyl-tRNA synthetaseinteracting multifunctional proteins (AIMPs): a triad for cellular homeostasis. IUBMB Life 62, 296-302. doi: 10.1002/iub.324

Park, S. G., Kang, Y. S., Ahn, Y. H., Lee, S. H., Kim, K. R., Kim, K. W., et al. (2002). Dose-dependent biphasic activity of tRNA synthetase-associating factor, p43, in angiogenesis. J. Biol. Chem. 277, 45243-45248. doi: 10.1074/jbc.M207934200

Pirone, L., Xolalpa, W., Sigurethsson, J. O., Ramirez, J., Perez, C., Gonzalez, M., et al. (2017). A comprehensive platform for the analysis of ubiquitin-like protein modifications using in vivo biotinylation. Sci. Rep. 7:40756. doi: 10.1038/ srep40756

Rubio Gomez, M. A., and Ibba, M. (2020). Aminoacyl-tRNA synthetases. RNA 26, 910-936. doi: 10.1261/rna.071720.119

Sampath, P., Mazumder, B., Seshadri, V., and Fox, P. L. (2003). Transcript-selective translational silencing by gamma interferon is directed by a novel structural element in the ceruloplasmin mRNA 3' untranslated region. Mol. Cell. Biol. 23, 1509-1519. doi: 10.1128/MCB.23.5.1509-1519.2003

Sampath, P., Mazumder, B., Seshadri, V., Gerber, C. A., Chavatte, L., Kinter, M., et al. (2004). Noncanonical function of glutamyl-prolyl-tRNA synthetase: genespecific silencing of translation. Cell 119, 195-208. doi: 10.1016/j.cell.2004.09. 030

Schimmel, P. R., and Soll, D. (1979). Aminoacyl-tRNA synthetases: general features and recognition of transfer RNAs. Annu. Rev. Biochem. 48, 601-648. doi: 10.1146/annurev.bi.48.070179.003125

Tatham, M. H., Matic, I., Mann, M., and Hay, R. T. (2011). Comparative proteomic analysis identifies a role for SUMO in protein quality control. Sci. Signal. 4:rs4. doi: 10.1126/scisignal.2001484

Waterhouse, A., Bertoni, M., Bienert, S., Studer, G., Tauriello, G., Gumienny, R., et al. (2018). SWISS-MODEL: homology modelling of protein structures and complexes. Nucleic Acids Res. 46, W296-W303. doi: 10.1093/nar/gk y427

Wolf, N. I., Salomons, G. S., Rodenburg, R. J., Pouwels, P. J., Schieving, J. H., Derks, T. G., et al. (2014). Mutations in RARS cause hypomyelination. Ann. Neurol. 76, 134-139. doi: 10.1002/ana.24167

Wolfe, C. L., Warrington, J. A., Davis, S., Green, S., and Norcum, M. T. (2003). Isolation and characterization of human nuclear and cytosolic multisynthetase complexes and the intracellular distribution of p43/EMAPII. Protein Sci. 12, 2282-2290. doi: 10.1110/ps.0314 7903

Yao, P., Poruri, K., Martinis, S. A., and Fox, P. L. (2014). Non-catalytic regulation of gene expression by aminoacyl-tRNA synthetases. Biol. Med. 344, 167-187. doi: 10.1007/128_2013_422

Zhang, Y., Werling, U., and Edelmann, W. (2014). Seamless ligation cloning extract (SLiCE) cloning method. Methods Mol. Biol. 1116, 235-244. doi: 10.1007/9781-62703-764-8_16

Conflict of Interest: The authors declare that the research was conducted in the absence of any commercial or financial relationships that could be construed as a potential conflict of interest.

Publisher's Note: All claims expressed in this article are solely those of the authors and do not necessarily represent those of their affiliated organizations, or those of the publisher, the editors and the reviewers. Any product that may be evaluated in this article, or claim that may be made by its manufacturer, is not guaranteed or endorsed by the publisher.

Copyright (c) 2021 Nayak, Kejriwal and Ratnaparkhi. This is an open-access article distributed under the terms of the Creative Commons Attribution License (CC BY). The use, distribution or reproduction in other forums is permitted, provided the original author(s) and the copyright owner(s) are credited and that the original publication in this journal is cited, in accordance with accepted academic practice. No use, distribution or reproduction is permitted which does not comply with these terms. 